\title{
Optimal Risk-Sensitive Filtering for System Stochastic of Second and Third Degree
}

\author{
Ma Aracelia Alcorta-Garcia, Sonia Gpe Anguiano Rostro, Mauricio Torres Torres \\ Department of Physical and Mathematical Sciences, Autonomous University of Nuevo Leon, Cd. Universitaria, \\ San Nicolas, Mexico \\ E-mail:aalcorta@fcfm.uanl.mx,srostro@hotmail.com,mautor2@gmail.com \\ Received October 9, 2010; revised February 8, 2011; accepted February 10, 2011
}

\begin{abstract}
The risk-sensitive filtering design problem with respect to the exponential mean-square cost criterion is considered for stochastic Gaussian systems with polynomial of second and third degree drift terms and intensity parameters multiplying diffusion terms in the state and observations equations. The closed-form optimal filtering equations are obtained using quadratic value functions as solutions to the corresponding FockerPlank-Kolmogorov equation. The performance of the obtained risk-sensitive filtering equations for stochastic polynomial systems of second and third degree is verified in a numerical example against the optimal polynomial filtering equations (and extended Kalman-Bucy for system polynomial of second degree), through comparing the exponential mean-square cost criterion values. The simulation results reveal strong advantages in favor of the designed risk-sensitive equations for some values of the intensity parameters.
\end{abstract}

Keywords: Optimal Nonlinear Filtering, Risk-Sensitive Filtering, Extended Kalman-Bucy Filtering

\section{Introduction}

Since the linear optimal filter was obtained by Kalman and Bucy (60's), numerous works are based on it, see for example [1-5], of the variety of all those. The deterministic filter model introduced by Mortensen [6] provides an alternative to stochastic filtering theory. In this model, errors in the state dynamics and the observations are modeled as deterministic "disturbance functions", and an exponential mean-square cost criterion disturbance error is to be minimized. Special conditions are given for the existence, continuity and boundedness of $f(X(t))$ in the state equation, which is considered nonlinear, and the linear function $h(X(t))$ in the observation equation. A concept of stochastic risk-sensitive estimator, introduced more recently by McEneaney [7], regard a dynamic system where $f(X(t))$ is a nonlinear function, linear observations and existence of parameter $\tilde{\varepsilon}$ multiplying the diffusion term in both equations (state and observations). In [8] were obtained the suboptimal risk-sensitive filtering equations for polynomial systems of third degree and applied to the pendulum equations [9], in which the original system was linearized applying Taylor series around the equilibrium point. In $[10,11]$ it is regarded $f(X(t))$ as nonlinear function. This paper presents an application of the equations obtained in $[10,11]$ for singular form of $f(X(t))$ (polynomial of second and third degree).

The goal of this work is to obtain the optimal risksensitive filtering equations when the form of $f(X(t))$ is polynomial of second and third degree and parameter $\tilde{\varepsilon}$ multiplying the diffusion term in the state and observations equations. There filtering equations are obtained taking a value function as solution of the nonlinear parabolic partial differential equation and exponential mean-square exponential cost criterion to be minimized.

Undefined parameters in the value function are calculated through ordinary differential equations composed by collecting terms corresponding to each power of the state-dependent polynomial in the nonlinear parabolic PDE equations. This procedure leads to the obtention of the optimal risk-sensitive filtering equations.

The closed-form for risk-sensitive filtering equations is explicitly obtained in this work. Although the difficulty presented by systems of second and third degree, in this work is shown an advantage for risk-sensitive filtering equations versus extended Kalman-Bucy and polynomial filtering equations under certain values of the parameter $\varepsilon$. This performance is shown verified in a numerical example against the mean-square optimal for 
polynomial filtering equations (and extended KalmanBucy for systems of second degree), through comparing the exponential mean-square cost criterion values in finite horizon time. The simulation results reveal strong advantages in favor of the designed risk-sensitive filtering equations for all values of the intensity parameters (in Table 1) multiplying diffusion terms in state and observation equations. Tables of the criterion values and graphs of the simulations are included. This exponential mean-square cost criterion function contains the parameter $\varepsilon$ which appear in the dynamic system, which leads to a more robust solution. This work is organized as follows: filtering problem statement, optimal risk-sensitive filtering for stochastic system of second degree, optimal risk-sensitive filtering for stochastic system of third degree, application for systems of second degree, application for systems of third degree, conclusions and references.

\section{Filtering Problem Statement}

Consider the following stochastic model (1), where $X(t)$ denotes the state process, $Y(t)$ denotes a continuous accumulated observations process, $X(t)$ satisfies the diffusion model given by

$$
d X(t)=f(X(t)) d t+\sqrt{\frac{\varepsilon}{2 \gamma^{2}}} d W(t)
$$

where $f(X(t))$ represents the nominal dynamics, and $W$ is a Brownian motion, and the observation process $Y(t)$ satisfies the equation:

$$
d Y(t)=h(X(t)) d t+\sqrt{\frac{\varepsilon}{2 \gamma^{2}}} d \tilde{W}(t), \quad Y(0)=0,
$$

where $\varepsilon$ is a parameter and $W$ and $\tilde{W}$ are independent Brownian motions, which are also independent of the initial state $X(0) . X(0)$ has probability density $k_{\varepsilon} \exp \left(-\varepsilon^{-1} \varphi(X(0))\right)$ for some constant $k_{\varepsilon}$.

Let us consider

$$
J=\varepsilon \log E\left[\exp \frac{1}{\varepsilon} \int_{0}^{T} L(X(t), m(t), t) d t / Y(t)\right]
$$

the exponential mean-square cost criterion to be minimize. In the rest of the paper the assumptions (A1)-(A4) (from [10]) are hold:

- (A1) $f, g, h \in \mathbb{R}^{n}$ with $f_{x}, h_{x}$ bounded.

- (A2) $D_{1}\left(|x|^{2}-1\right) \leq \phi(x) \leq D_{2}\left(|x|^{2}+1\right)$. Here $f_{x}$ is the matrix of partial derivatives of $f$ with $h_{x}$ defined similarly. $\phi(x)$ is a continuous, real-valued function satisfying (A2) for some positive $D_{1}, D_{2}$.

- (A3) $f, h \in \mathbb{R}^{n}$ with $f, h$ bounded and $f_{x x}, h_{x x}$ bounded and globally Hölder continuous. (A function $u$ is globally
Hölder continuous if there exists $\alpha \in(0,1], K<\infty$ such that $|u(x)-u(y)| \leq K|x-y|^{\alpha}$ for all $\left.x, y\right)$.

- (A4) Given $R<\infty$, there exists $K_{R}<\infty$ such that $|\phi(x)-\phi(y)| \leq K_{R}|x-y|$ for all $|x|,|y|<\infty$.

Let $q(T, X(t))$ denotes the unnormalized conditional density of $X(T)$, given accumulated observations $Y(t)$ for $0 \leq t \leq T$. It satisfies the Zakai stochastic PDE, in a sense made precise, for instance in [12]. It is assumed that

$$
\begin{aligned}
& q(0, X(t))=\exp \left(-\varepsilon^{-1} \varphi(X(t))\right), \\
& q(T, X(t))=p(T, X(t)) \exp \left[-\varepsilon^{-1} Y(T) \cdot h(x(t))\right],
\end{aligned}
$$

where $p(T, X(t))$ is called pathwise unnormalized filter density. $p$ satisfies the following linear secondorder parabolic PDE with coefficients depending on $Y(t)$ :

$$
\frac{\partial p}{\partial T}=(\breve{L}(T))^{*} p+\frac{K}{\varepsilon} p .
$$

where, for every $g \in \mathbb{R}^{n}$, let

$$
\begin{aligned}
& L_{g}=\frac{\varepsilon}{2} \operatorname{tr}\left(a g_{X X}\right)+f \cdot g_{X}, \\
& \breve{L}(T) g=L g-a(Y(T) \cdot h)_{X} \cdot g_{X} \\
& K(T, X(t))=\frac{1}{2} a(X(t))(Y(T) \cdot h)_{X} \cdot(Y(T) \cdot h)_{X} \\
& -L(Y(T) \cdot h)-\frac{1}{2}|h|^{2}
\end{aligned}
$$

$L$ denotes the differential generator of the Markov diffusion $X(t)$ in (1). By assumptions (A1) and (A3) in [10], $K$ is bounded and continuous. $(\breve{L}(T))^{*}$ is the formal adjoint of $\breve{L}(T)$. Since $Y(0)=0, \quad p(0, X(t))$ $=q(0, X(t))$. The initial condition for (5) is (4). For some given $Y \in C_{0}(0, T]$, (where $C_{0}$ denote the space of continuous $Y(t)$ such that $Y(0)=0$, with the sup norm \|\|$)$. The pathwise filter density $p$ is the unique "strong" solution to (5) and (4) in a sense made precise in [12]. Further, $p$ is a classical solution to (5) and (4) with $p$ continuous on $\left[0, T_{1}\right] \times \mathbb{R}^{n}$ and partial derivatives $p_{T}, p_{X_{i}}, p_{X_{i} X_{j}}, i, j=1, \cdots, n$ continuous for $0<T \leq T_{1}$ $[13,14]$.

Moreover, $p(T, X(t) ; Y)>0$. We rewrite (5) as follows:

$$
\frac{\partial p}{\partial T}=\frac{1}{2} \operatorname{tr}\left(a(X(t)) p_{X X}\right)+A \cdot p_{X}+\frac{B}{\varepsilon} p
$$

where

$$
\begin{aligned}
A= & -f(X(t))+a(X(t))(Y(t) \cdot h(x(t)))_{X} \\
& +\varepsilon \operatorname{div}\left(a_{X}(t)\right) \\
B= & (T, X(t))
\end{aligned}
$$




$$
\begin{aligned}
& =\frac{\varepsilon^{2}}{2} \operatorname{tr}\left(a_{X X}(X(t))\right) \\
& \quad-\varepsilon \operatorname{div}\left[f(X(t))-a(X(t))(Y(T) \cdot h(X(t)))_{X}\right] \\
& \quad+K(T, X(t)), \\
& \left(\operatorname{div}\left(a_{X}(t)\right)\right)_{j}=\sum_{i, j=1}^{n}\left(a_{i j}\right)_{X_{i}}, j=1, \cdots, n \\
& \operatorname{tr}\left(a_{X X}\right)=\sum_{i, j=1}^{n}\left(a_{i j}\right)_{X_{i} X_{j}}
\end{aligned}
$$

These assumptions imply uniform bounds for $A$ and $B$, depending on the sup norm $\|Y\|$ on $\left[0, T_{1}\right]$, but not on $\varepsilon$. Taking log transform: $Z(T, X(t))=\varepsilon \log p(T, X(t))$, which satisfies the nonlinear parabolic PDE:

$$
\frac{\partial Z}{\partial T}=\frac{\varepsilon}{2} \operatorname{tr}\left(Z_{X X}\right)+A \cdot Z_{X}+\frac{1}{2} Z_{X} \cdot Z_{X}+B,
$$

with initial condition $Z_{X}(0, X(t))=-\varphi(X(t))$. The optimal risk-sensitive filtering problem consists in found the estimate $C(t)$, of the state $X(t)$ through verification that

$$
\begin{aligned}
Z(T, X(t)) & =\frac{1}{2}(X(t)-C(t))^{T} Q(t)(X(t)-C(t)) \\
& +\rho(T)-Y(T) \cdot h(X(t))
\end{aligned}
$$

is a viscosity solution of (9).

Where $X(t) \in \mathbb{R}^{n}, \quad w(t) \in \mathbb{R}^{m}, \quad Y(t), \quad v(t) \in \mathbb{R}^{p}$, $f, h \in \mathbb{R}^{n}$ with $f_{X}, h_{x}$ bounded is assumed throughout. Here $h_{x}$ is the matrix of partial derivatives of $h$ and the same form for $Z_{X}$.

\section{Optimal Risk-Sensitive Filtering Problem}

\subsection{Optimal Risk-Sensitive Filtering for Stochastic System of Second Degree}

Taking $f(X(t))=A(t)+A_{1}(t) X(t)+A_{2}(t) \cdot X^{T}(t) X(t)$, $h(X(t))=E(t)+E_{1}(t) X(t)$ with $A(t) \in \mathbb{R}^{n}, A_{1}(t) \in M_{n \times n}$, $A_{2}(t) \in T_{n \times n \times n}, \quad E(t) \in E_{1}(t) \in \mathbb{R}^{p}, \quad M_{n \times p}$ where $M_{i \times j}$ denotes the field of matrixes of dimension $i \times j$ and $T_{i \times j \times k}$ denotes the field of tensors of dimension $i \times j \times k \quad$ n. The following stochastic equations system is obtained:

$$
\begin{aligned}
d X(\mathrm{t})= & A(t)+A_{1}(t) X(t)+A_{2}(t) X^{T}(t) X(t) \\
& +\sqrt{\tilde{\varepsilon}} d B(t) \\
d Y(t)= & E(t)+E_{1}(t) X(t)+\sqrt{\tilde{\varepsilon}} d \tilde{B}(t)
\end{aligned}
$$

where $\tilde{\varepsilon}=\varepsilon /\left(2 \gamma^{2}\right)$. The optimal filtering problem consists in to obtain the estimate of the state $X(t)$ given the observations equations, which minimizes the expo-

nential mean-square cost criterion, taking $Z(T, X(t))$ $(10)$ as solution of the nonlinear parabolic partial differential Equation (9).

Theorem 1. The solution to the filtering problem, for the system (11) with criterion (3) takes the form:

$$
\begin{aligned}
\dot{C}(t)= & A(t)+A_{1}(t) C(t)-\dot{Q}(t) Q^{-1}(t) C(t) \\
& +Q^{-1}(t) \cdot E_{1}(t)(d y-E(t))+Q(t) C(t) \\
& +2 \varepsilon A_{2}(t) Q^{-1}(t), \\
\dot{Q}(t)= & -A_{1}(t) Q(t)-Q(t) A_{1}^{T}(t)+Q^{T}(t) Q(t) \\
& -E_{1}^{T}(t) E_{1}(t) .
\end{aligned}
$$

where $C(t)$ is the state estimate vector with initial conditions with initial condition $C(0)=C_{0}$, and $Q(t)$ is a symmetric matrix negative defined, where the initial condition $Q(0)=q_{0}$ is derived from initial conditions for Z. If $\varphi(X(t))=X^{T}(t) K X(t), Q(0)=-K$.

Proof: The value function is proposed

$$
\begin{aligned}
Z(T, X(t)) & =\frac{1}{2}(X(t)-C(t))^{T} Q(t)(X(t)-C(t)) \\
& +\rho(T)-Y(T) \cdot h(X(t)), \\
Z_{X}(0, X(t)) & =-\varphi(X(t)),(C(t), Q(t), \rho(t)) \text { are func- }
\end{aligned}
$$
tions defined on $[0, T], C(t) \in \mathbb{R}^{n}, Q(t)$ is a symmetric matrix of dimension $n \times n$ and $\rho(t)$ is a scalar function) as a viscosity solution of the nonlinear parabolic PDE (9). $Z_{X}, Z_{X X}$ are the partial derivatives of $Z$ respect to $X(t)$ and $\nabla Z$ is the gradient of $Z$. Then the partial derivatives of $Z$ are given by:

$$
\begin{aligned}
Z_{T}= & \frac{1}{2}(X(t)-C(t))^{T} \dot{Q}(X(t)-C(t)) \\
& -(X(t)-C(t))^{T} Q(t) \dot{C}(t)+\dot{\rho}(t) \\
& -\dot{Y}(t)\left(E(t)-E_{1}(t) X(t)\right) \\
Z_{X}= & \frac{1}{2} Q(t)(X(t)-C(t)) \\
& +\frac{1}{2}(X(t)-C(t))^{T} Q(t)-Y(t) E_{1}(t), \\
Z_{X X} & =Q(t) .
\end{aligned}
$$

Let us consider:

$$
\begin{aligned}
A= & -A(t)-A_{1}(t) X(t)-A_{2}(t) X^{T}(t) X(t) \\
& +Y(T) E_{1}(t) \\
B= & -2 \varepsilon A_{2}(t) X(t)-\varepsilon A_{1}(t)+\frac{1}{2}\left(Y(T) E_{1}(t)\right)^{2} \\
& \times\left(-A(t)-A_{1}(t) X(t)-A_{2}(t) X^{T}(t) X(t)\right. \\
& \left.+Y(T) E_{1}(t)\right)-\frac{1}{2}\left|E(t)+E_{1}(t) X(t)\right|^{2}
\end{aligned}
$$


Substituting (14) and the expressions for A, B in (9); we obtain:

$$
\begin{aligned}
0= & -\frac{1}{2}(X(t)-C(t))^{T} \dot{Q}(t)(X(t)-C(t))^{T} Q(t) \dot{C}(t) \\
& -\dot{\rho}(t)+\dot{Y}(t)\left(E(t)+E_{1}(t) X(t)\right) \frac{\varepsilon}{2} \operatorname{tr}(Q(t)) \\
& +\left(-A(t)-A_{1}(t) X(t)-A_{2}(t) X^{T}(t) X(t)+Y(t)\right. \\
& \left.\times E_{1}(t)\right)\left(\frac{1}{2} Q(t) \cdot(X(t)-C(t))+\frac{1}{2}(X(t)-C(t))\right)^{T} \\
& \times Q(t)-Y(t) E_{1}(t)+\frac{1}{2}\left(\frac{1}{2} Q(t)(X(t)-C(t))\right. \\
& \left.+\frac{1}{2}(X(t)-C(t))^{T} Q(t)-Y(t) E_{1}(t)\right)-\varepsilon A_{1}(t) \\
& +\frac{1}{2}\left(Y(t) E_{1}(t)\right)^{2}\left(-A(t)-A_{1}(t) X(t)-A_{2}(t) X^{T}(t)\right. \\
& \left.\times X(t)+Y(t) E_{1}(t)\right)-\frac{1}{2}\left|E(t)+E_{1}(t) X(t)\right|^{2} .
\end{aligned}
$$

Collecting the $X^{T}(t) X(t), X^{T}(t) X(t) X^{T}(t)$ and $X^{T}(t) X(t) X^{T}(t) X(t)$ terms, and replacing $X(t)$ by $C(t)$; we obtain the matrix equation for $\dot{Q}(t)$. Collecting the $X(t)$ terms, the vectorial equations for $\dot{C}(t)$ are obtained (12).

\subsection{Optimal Risk-Sensitive Filtering for Stochastic System of Third Degree}

Taking $f(X(t))=A(t)+A_{1}(t) X(t)+A_{2}(t) X^{T}(t) \cdot X(t)$ $+A_{3}(t) X^{T}(t) X(t) X^{T}(t), h(X(t))=E(t)+E_{1}(t) X(t)$ with $A(t) \in \mathbb{R}^{n}, A(t) \in M_{n \times n}, A_{2}(t) \in T_{n \times n \times n}, A_{3}(t) \in T_{n \times n \times n \times n}$, $E(t) \in \mathbb{R}^{p}, E_{1}(t) \in M_{n \times p}$ where $M_{i \times j}$ denotes the field of matrixes of dimension $i \times j, T_{i \times j \times k}$ denotes the field of tensors of dimension $i \times j \times k$ and $T_{i \times j \times k \times l}$ denotes the field of tensors of dimension $i \times j \times k \times l$. The following stochastic equations system is obtained:

$$
\begin{aligned}
d X(\mathrm{t})= & \mathrm{A}(t)+A_{1}(t) X(t)+A_{2}(t) X^{T}(t) X(t) \\
& +A_{3}(t) X^{T}(t) X(t) X^{T}(t)+\sqrt{\tilde{\varepsilon}} d B(t), \\
d Y(t)= & E(t)+E_{1}(t) X(t)+\sqrt{\tilde{\varepsilon}} d \tilde{B}(t), X(0)=X_{0},
\end{aligned}
$$

where $\tilde{\varepsilon}=\varepsilon /\left(2 \gamma^{2}\right)$.

Theorem 2. The solution to the filtering problem, for the system (17) with criterion (3) takes the form:

$$
\begin{aligned}
\dot{C}(t) & =A(t)-A_{1}(t) C(t)-\dot{Q}(t) Q^{-1}(t) C(t) \\
& -Q^{-1}(t) \cdot E_{1}(t)\left(d y-\frac{1}{2} E(t)\right)+Q(t) C(t), \\
\dot{Q}(t)= & A_{1}(t) Q(t)+Q(t) A_{1}^{T}(t)-A_{2}(t) Q(t) C(t) \\
& -\left(A_{2}(t) Q(t) C(t)\right)^{T}+A_{2}(t) Q(t) C(t)
\end{aligned}
$$

$$
\begin{aligned}
& -A_{3}(t) C(t) C^{T}(t) Q(t) \\
& -\left(A_{3}(t) C(t) C^{T}(t) Q(t)\right)^{T} \\
& +A_{3}(t) Q(t) C(t) C^{T}(t) \\
& +\left(A_{3}(t) Q(t) C(t) C^{T}(t)\right)^{T} \\
& +Q^{T}(t) Q(t)-\varepsilon \operatorname{div}\left[f(C)-Y(t) E_{1}(t)\right] \\
& -E_{1}^{T}(t) E_{1}(t)
\end{aligned}
$$

where $C(t)$ is the state estimate vector with initial conditions with initial condition $C(0)=C_{0}$, and $Q(t)$ is a symmetric matrix negative defined, where the initial condition $Q(0)=q_{0}$ is derived from initial conditions for Z. If $\varphi(X(t))=X^{T}(t) K X(t), Q(0)=-K$.

Proof: In similar form to Theorem 1.

\section{Applications}

\subsection{Application for Systems of Second-Degree. Optimal Risk-Sensitive Filtering Equations}

Consider the following dynamical stochastic system associated to a continuous stirred tank reactor in which is a chemical reaction occurs. This reaction is in liquid phase and has isothermal character between multicomponents [15].

$$
\begin{aligned}
& \dot{X}_{1}(t)=-\left(1+D_{a 1}\right) X_{1}+u(t)+\sqrt{\frac{\varepsilon}{2 \gamma^{2}}} d W_{1}(t), \\
& \dot{X}_{2}(t)=D_{a 1} X_{1}-X_{2}-D_{a 2} X_{2}^{2}+\sqrt{\frac{\varepsilon}{2 \gamma^{2}}} d W_{2}(t) .
\end{aligned}
$$

where $X_{1}(t)$ represents the unnormalized concentration $P / C_{P_{o}}$ of a certain specie $P$ of the reactor, $X_{2}(t)$ represents unnormalized concentration $C_{Q} / C_{P_{o}}$ of a certain specie $Q$. The control variable $u$ is defined as the relation between the alimentation molar rate by volumetric unit of $P$, designated by $N_{P F}$ and the nominal concentration $C_{P o}$, this is $u=N_{P F} / F C_{P_{o}}$, where $F$ is the volumetric flow of alimentation on $\mathrm{m}^{3} \mathrm{~s}^{-1} . D_{a 1}=k_{1} V / F$, $D_{a 2}=k_{2} V C_{P_{0}} / F$ where $V$ is the volume of reactor in $\mathrm{m}^{3}, k_{1}$ and $k_{2}$ are constants of first degree given in $\mathrm{s}^{-1}$. It can take that $D_{a 1}$ and $D_{a 2}$ are considering by $D_{a 1}=1$ and $D_{a 2}=1 . Q$ is highly sour while $P$ is neuter. Then, the following dynamical stochastic system is obtained:

$$
\begin{aligned}
& \dot{X}_{1}(t)=-2 X_{1}+u(t)+\sqrt{\frac{\varepsilon}{2 \gamma^{2}}} d W_{1}(t), \\
& \dot{X}_{2}(t)=X_{1}-X_{2}-X_{2}^{2}+\sqrt{\frac{\varepsilon}{2 \gamma^{2}}} d W_{2}(t) .
\end{aligned}
$$


Applying the equations (12) to the system (20), the equations of the optimal risk-sensitive filtering are obtained:

$$
\begin{aligned}
\dot{Q}_{11}= & 4 Q_{11}+Q_{11}^{2} Q_{12}^{2}-1, \\
\dot{Q}_{12}= & 3 Q_{12}+Q_{11}+Q_{11} Q_{12}-Q_{12} Q_{22}, \\
\dot{Q}_{22} & =2 Q_{22}-2 Q_{12}+Q_{12}^{2}+Q_{22}^{2}-1, \\
\dot{C}_{1}= & \frac{-\dot{Q}_{11}}{Q_{11} Q_{22}-Q_{12}^{2}}\left[\dot{Q}_{11}\left(Q_{22} C_{1}-Q_{12} C_{2}\right)\right. \\
& \left.+\dot{Q}_{12}\left(Q_{11} C_{2}-Q_{12} C_{1}\right)-\dot{Y}_{1} Q_{22}+\dot{Y}_{2} Q_{12}\right] \\
& -2 C_{1}+Q_{11} C_{1}+Q_{12} C_{2}, \\
\dot{C}_{2}= & \frac{-\dot{Q}_{12}}{Q_{11} Q_{22}-Q_{12}^{2}}\left[\dot{Q}_{12}\left(Q_{22} C_{1}-Q_{12} C_{2}\right)\right. \\
& \left.+\dot{Q}_{22}\left(Q_{11} C_{2}-Q_{12} C_{1}\right)-\dot{Y}_{1} Q_{12}+\dot{Y}_{2} Q_{11}-2 \varepsilon Q_{11}\right] \\
& +C_{1}-C_{2}+Q_{12} C_{1}+Q_{22} C_{2} .
\end{aligned}
$$

The initial conditions for the risk-sensitive filtering equations are: $X_{1}(0)=20, X_{2}(0)=10, Y_{1}(0)=2, Y_{2}(0)=1$, $C_{1}(0)=1, \quad C_{2}(0)=5, \quad Q_{11}(0)=6, \quad Q_{12}(0)=0.0001$, $Q_{22}(0)=-7$, the final time is $T=2 \mathrm{~s}$. The system formed by the equations (20) and (21), is simulated using Simulink in MatLab7. The performance of the designed equations is compared versus the equations of the polynomial filtering [1] and the equations of the extended Kalman-Bucy filtering [16], applied to the system (20), that is optimal with respect to the conventional exponential mean-square cost criterion.

\subsubsection{Polynomial Filtering Equations}

The corresponding equations for the polynomial filtering [1] are given by:

$$
\begin{aligned}
\dot{P}_{11}= & 4 P_{11}+\frac{\varepsilon}{2 \gamma^{2}}-\frac{2 \gamma^{2}}{\varepsilon}\left(P_{12}^{2}+P_{11}^{2}\right), \\
\dot{P}_{12}= & P_{11}-3 P_{12}-2 m_{2} P_{12}-\frac{2 \gamma^{2}}{\varepsilon}\left(P_{11} P_{12}+P_{12} P_{22}\right), \\
\dot{P}_{22}= & 2 P_{22}+2 P_{12}-4 m_{2} P_{22}+\frac{\varepsilon}{2 \gamma^{2}}-\frac{2 \gamma^{2}}{\varepsilon}\left(P_{12}^{2}+P_{22}^{2}\right), \\
\dot{m}_{1}= & -2 m_{1}+\frac{2 \gamma^{2}}{\varepsilon}\left[P_{11}\left(\dot{Y}_{1}-m_{1}\right)+P_{12}\left(\dot{Y}_{2}-m_{2}\right)\right], \\
\dot{m}_{2}= & m_{1}-m_{1}-m_{2}^{2}-P_{22} \\
& +\frac{2 \gamma^{2}}{\varepsilon}\left[P_{12}\left(\dot{Y}_{1}-m_{1}\right)+P_{22}\left(\dot{Y}_{2}-m_{2}\right)\right] .
\end{aligned}
$$

where the initial conditions are $X_{1}(0)=20, X_{2}(0)=10$, $Y_{1}(0)=2, Y_{2}(0)=1, m_{1}(0)=1, P_{11}(0)=100, P_{12}(0)=-1$, $P_{22}(0)=1 \times 10^{7}$.

\subsubsection{Extended Kalman-Bucy Filtering Equations}

The equations of the extended Kalman-Bucy [13] filtering are given by:

$$
\begin{aligned}
& \dot{P}_{11}=4 P+\frac{\varepsilon}{2 \gamma^{2}}-\frac{2 \gamma^{2}}{\varepsilon}\left(P_{12}^{2}+P_{11}^{2}\right), \\
& \dot{P}_{12}=P_{11}-3 P_{12}-\frac{2 \gamma^{2}}{\varepsilon}\left(P_{11} P_{12}+P_{12} P_{22}\right), \\
& \dot{P}_{22}=2 P_{12}-2 P_{22}+\frac{\varepsilon}{2 \gamma^{2}}-\frac{2 \gamma^{2}}{\varepsilon}\left(P_{12}^{2}+P_{22}^{2}\right), \\
& \dot{m}_{1}=-2 m_{1}+\frac{2 \gamma^{2}}{\varepsilon}\left[P_{11}\left(\dot{Y}_{1}-m_{1}\right)+P_{12}\left(\dot{Y}_{2}-m_{2}\right)\right], \\
& \dot{m}_{2}=m_{1}-m_{1}+\frac{2 \gamma^{2}}{\varepsilon}\left[P_{12}\left(\dot{Y}_{1}-m_{1}\right)+P_{22}\left(\dot{Y}_{2}-m_{2}\right)\right] .
\end{aligned}
$$

1) Consider the stochastic dynamical system associated to a continuous stirred tank reactor and the following initial conditions for the state and observations equations: $X_{1}(0)=20, X_{2}(0)=10, Y_{1}(0)=2, Y_{2}(0)=1$, the final time is $T=2 \mathrm{~s}$. The initial conditions for the filtering equations in which case are given by:

a) For risk-sensitive filtering equations:

$$
\begin{aligned}
& C_{1}(0)=1, C_{2}(0)=5, Q_{11}(0)=6, Q_{12}(0)=0.0001, \\
& Q_{22}(0)=-7 .
\end{aligned}
$$

b) For polynomial filtering equations:

$$
\begin{aligned}
& m_{1}(0)=1, m_{2}(0)=5, P_{11}(0)=100, P_{12}(0)=-1, \\
& P_{22}(0)=1 \times 10^{7} .
\end{aligned}
$$

c) For Extended Kalman-Bucy filtering equations:

$$
\begin{aligned}
& m_{1}(0)=1, m_{2}(0)=5, P_{11}(0)=5, P_{12}(0)=3, \\
& P_{22}(0)=5 .
\end{aligned}
$$

Table 1 presents comparison between the exponential mean square cost criterion $J$ for the three types of filtering equations; you can see that the $J_{R-S}$ values are the smallest for all values of the intensity parameter $\varepsilon$.

2) Consider the stochastic dynamical system associated to a continuous stirred tank reactor and the following initial conditions for the state and observations equations: $X_{1}(0)=50, X_{2}(0)=1, Y_{1}(0)=2, Y_{2}(0)=1$, the final time is $T=2 \mathrm{~s}$. The initial conditions for the filtering equations in which case are given by:

a) For risk-sensitive filtering equations:

$$
\begin{aligned}
& C_{1}(0)=1, C_{2}(0)=5, Q_{11}(0)=-7, Q_{12}(0)=0.0001, \\
& Q_{22}(0)=-7.5 \text {. }
\end{aligned}
$$

b) For polynomial filtering equations:

$$
\begin{aligned}
& m_{1}(0)=1, m_{2}(0)=5, P_{11}(0)=85, P_{12}(0)=-10, \\
& P_{22}(0)=2 \times 10^{7} .
\end{aligned}
$$

c) For Extended Kalman-Bucy filtering equations:

$$
\begin{aligned}
& m_{1}(0)=1, m_{2}(0)=5, P_{11}(0)=2, P_{12}(0)=5, \\
& P_{22}(0)=10 .
\end{aligned}
$$


Table 2 presents comparison between the exponential mean square cost criterion $J$ for the three types of filtering equations; you can see that the $J_{R-S}$ values are the smallest for all values of the intensity parameter $\varepsilon$.

3) Consider the stochastic dynamical system associated to a continuous stirred tank reactor and the following initial conditions for the state and observations equations: $X_{1}(0)=0.05, X_{2}(0)=50, Y_{1}(0)=2, Y_{2}(0)=1$, the final time is $T=2 \mathrm{~s}$. The initial conditions for the filtering equations in which case are given by:

a) For risk-sensitive filtering equations:

$$
\begin{aligned}
& C_{1}(0)=1, C_{2}(0)=5, Q_{11}(0)=-6, Q_{12}(0)=0.0001, \\
& Q_{22}(0)=-7
\end{aligned}
$$

b) For polynomial filtering equations:

$$
\begin{aligned}
& m_{1}(0)=1, m_{2}(0)=5, P_{11}(0)=100, P_{12}(0)=-5, \\
& P_{22}(0)=1 \times 10^{7} .
\end{aligned}
$$

c) For Extended Kalman-Bucy filtering equations:

$$
\begin{aligned}
& m_{1}(0)=1, m_{2}(0)=5, P_{11}(0)=1.85, P_{12}(0)=3, \\
& P_{22}(0)=5 .
\end{aligned}
$$

Table 3 presents comparison between the exponential mean square cost criterion $J$ for the three types of filtering equations; you can see that the $J_{R-S}$ values are the smallest for all values of the intensity parameter $\varepsilon$.

With these tables, showed that the filter risk-sensitive is the best, because the values obtained are lower.

The Figures 1, 2 and $\mathbf{3}$ show the Error $_{1}$ and Error $_{2}$ which are defined as Error ${ }_{1}=X_{1}(t)-C_{1}(t)$ (in same form for Error $_{2}$ ); and the exponential mean-square cost criterion values in $T=2 \mathrm{~s}$.

Table 1. Comparison of exponential mean-square cost criterion values $J(3)$ in $T=2 s$ for risk-sensitive, polynomial and extended Kalman-Bucy filtering equations.

\begin{tabular}{cccc}
\hline$\varepsilon$ & $J_{R-S}$ & $J_{P_{O} l}$ & $J_{K-B}$ \\
\hline 0.1 & 53.4293 & $69.2292(t=0.17 \mathrm{~s})$ & $69.0816(t=0.14 \mathrm{~s})$ \\
1 & 53.5165 & 145.7323 & 277.3136 \\
10 & 53.7994 & 157.2172 & 235.5110 \\
100 & 54.7621 & 858.7622 & 189.6937 \\
1000 & 58.5054 & 58230 & 185.7343 \\
\hline
\end{tabular}

Table 2. Comparison of exponential mean-square cost criterion values $J(3)$ in $T=2 s$ for risk-sensitive, polynomial and extended Kalman-Bucy filtering equations.

\begin{tabular}{cccc}
\hline$\varepsilon$ & $J_{R-S}$ & $J_{P_{O l}}$ & $J_{K-B}$ \\
\hline 1 & 505.8493 & $705.1152(t=1.64 \mathrm{~s})$ & $686.3813(t=0.28 \mathrm{~s})$ \\
10 & 513.3591 & 712.2522 & 527.0787 \\
100 & 537.8120 & 1430.4728 & 587.0328 \\
1000 & 622.1946 & 59067 & 641.1202 \\
10000 & 960.423 & 5597700 & 673.6555 \\
\hline
\end{tabular}

Table 3. Comparison of exponential mean-square cost criterion values $J(3)$ in $T=2 s$ for risk-sensitive, polynomial and extended Kalman-Bucy filtering equations.

\begin{tabular}{cccc}
\hline$\varepsilon$ & $J_{R-S}$ & $J_{\text {Pol }}$ & $J_{K-B}$ \\
\hline 0.1 & 42.0377 & 55.6339 & $70.3916(t=0.36 \mathrm{~s})$ \\
1 & 41.9340 & 56.1153 & 144.2845 \\
10 & 41.6143 & 70.7942 & 317.9369 \\
100 & 40.6851 & 763.7829 & 678.2942 \\
1000 & 38.5611 & 57957 & 812.9141 \\
10000 & 36.1603 & 55918000 & 859.8006 \\
\hline
\end{tabular}
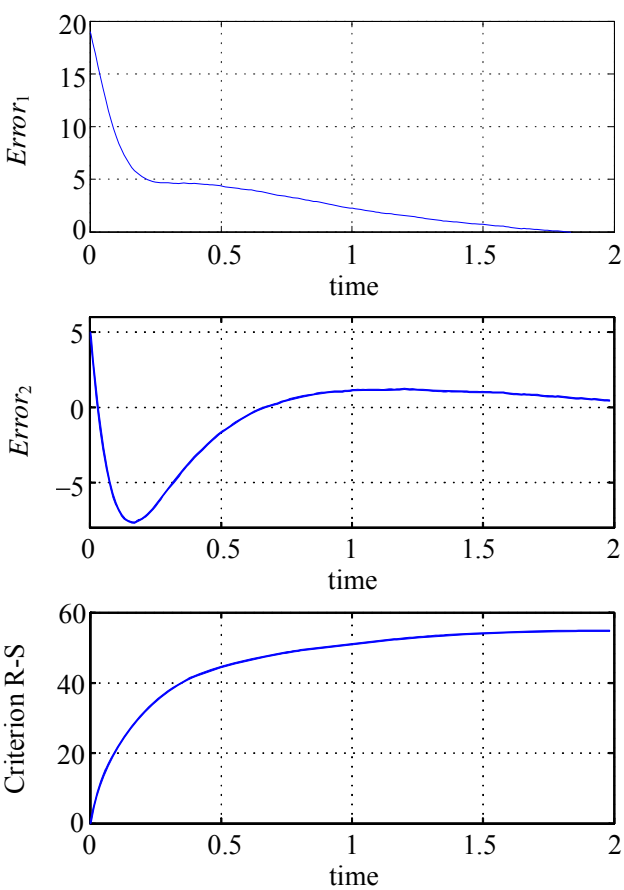

Figure 1. Graphs of the Error, Error $_{2}$, and exponential mean square cost criterion corresponding to the risk-sensitive optimal filtering equations for a continuous stirred tank reactor for $\varepsilon=10, X_{1}(0)=20, X_{2}(0)=10, Y_{1}(0)=2$, $Y_{2}(\mathbf{0})=1$.

\subsection{Application for Polynomial System of Third Degree}

\subsubsection{Optimal Risk-Sensitive Filtering Equations}

The risk-sensitive control equations for third degree polynomial systems will be applied to the problem of orientation of a monoaxial satellite [15]. The description is as follows: a satellite rotates around a fixed axis without gravity. The rotation torques is produced by a system of mini-engines through a controlled explosion of gases in the opposite direction. The state equations for this model are given by:

$$
\dot{X}_{1}(t)=0.5\left(1+X_{1}^{2}(t)\right) X_{2}(t)+\sqrt{\frac{\varepsilon}{2 \gamma^{2}}} d W_{1}(t)
$$




$$
\dot{X}_{2}(t)=\sqrt{\frac{\varepsilon}{2 \gamma^{2}}} d W_{2}(t), \dot{Y}(t)=X_{1}(t)+\sqrt{\frac{\varepsilon}{2 \gamma^{2}}} d W(t) .
$$

where $X_{1}(t)$ represents the orientation angle of the satellite, measured with respect of a secondary axis which does not coincide with the principal one. $X_{2}(t)$ represents the angular velocity with respect to the principal axis. Applying the system of equations (18) to the system (24), the following optimal risk-sensitive filtering equations are obtained:

$$
\begin{aligned}
\dot{Q}_{11}= & Q_{12}-C_{1} C_{2} Q_{11}-C_{2}^{2} Q_{12}+C_{2} Q_{22}+Q_{11} \\
& +Q_{22}-\varepsilon C_{1} C_{2}, \\
\dot{Q}_{12}= & 0.5 Q_{22}+Q_{11} Q_{12}+Q_{12} Q_{22}, \\
\dot{Q}_{22}= & Q_{12}^{2}+Q_{22}^{2}-\varepsilon C_{1} C_{2}, \\
\dot{C}_{1}= & \frac{-\dot{Q}_{22}}{Q_{11} Q_{22}-Q_{12}^{2}}\left(C_{1} \dot{Q}_{11}+C_{2} \dot{Q}_{12}+\dot{Y}\right) \\
& +\frac{-\dot{Q}_{12}}{Q_{11} Q_{22}-Q_{12}^{2}}\left(C_{1} \dot{Q}_{12}+C_{2} \dot{Q}_{22}\right)-\frac{1}{2} C_{2} \\
& +C_{1} Q_{11}+C_{2} Q_{12}, \\
\dot{C}_{2}= & \frac{-\dot{Q}_{12}}{Q_{11} Q_{22}-Q_{12}^{2}}\left(C_{1} \dot{Q}_{11}+C_{2} \dot{Q}_{12}+\dot{Y}\right) \\
& +\frac{-\dot{Q}_{11}}{Q_{11} Q_{22}-Q_{12}^{2}}\left(C_{1} \dot{Q}_{12}+C_{2} \dot{Q}_{22}\right)+C_{1} Q_{12} \\
& +C_{2} Q_{22} .
\end{aligned}
$$
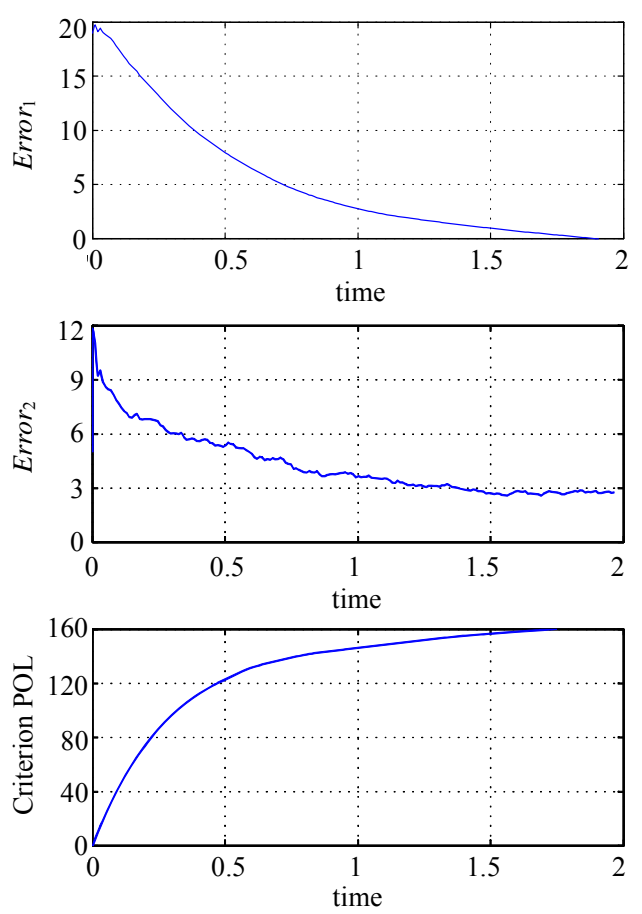

Figure 2. Graphs of the Error ${ }_{1}$, Error $_{2}$, and exponential mean square cost criterion corresponding to the polynomial filtering equations for a continuous stirred tank reactor for $\varepsilon=10, X_{1}(0)=20, X_{2}(0)=10, Y_{1}(0)=2, \quad Y_{2}(0)=1$.
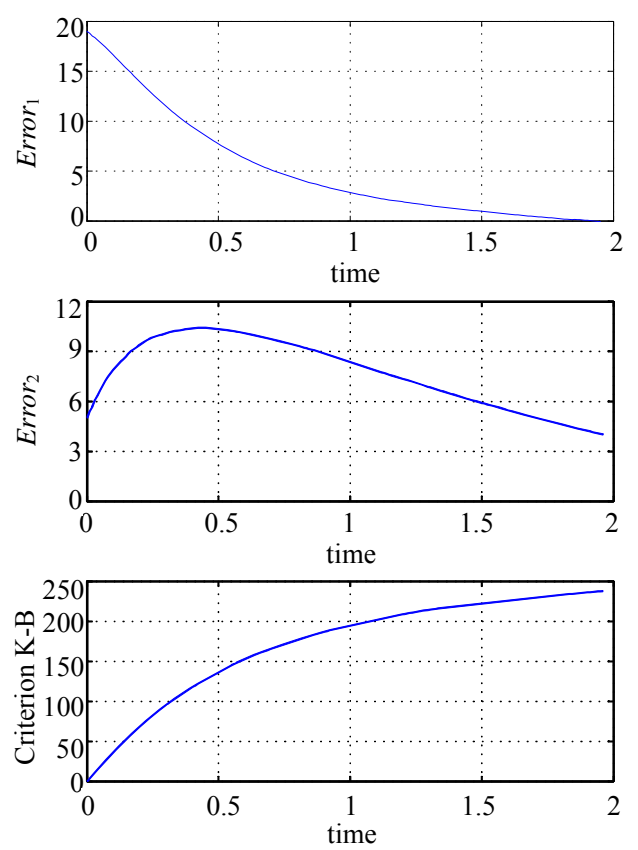

Figure 3. Graphs of the Error ${ }_{1}$, Error $_{2}$, and exponential mean square cost criterion corresponding to the extended Kalman-Bucy filtering equations for a continuous stirred tank reactor for $\varepsilon=10, X_{1}(0)=20, X_{2}(0)=10, Y_{1}(0)=2$, $Y_{2}(\mathbf{0})=1$.

The initial conditions are: $X_{1}(0)=0.115, X_{2}(0)=0.073$, $Y_{1}(0)=0, C_{1}(0)=0.92$, and $C_{2}(0)=0.5, Q_{11}(0)=-4500$, $Q_{12}(0)=100, \quad Q_{22}(0)=-8500, \quad T=1.8 \mathrm{~s}$.

\subsubsection{Polynomial Filtering Equations}

The corresponding equations for the polynomial filter [1] are given by:

$$
\begin{aligned}
\dot{P}_{11}= & P_{12}+3 P_{12} P_{11}+3 P_{12} P_{22}+3 P_{11} m_{1} m_{2} \\
& +3 P_{12} m_{2}^{2}+\frac{\varepsilon}{2 \gamma^{2}}-\frac{2 \gamma^{2} P_{11}^{2}}{\varepsilon}, \\
\dot{P}_{12}= & 0.5 P_{22}-\frac{2 \gamma^{2} P_{11} P_{12}}{\varepsilon}, \dot{P}_{22}=\frac{\varepsilon}{2 \gamma^{2}}-\frac{2 \gamma^{2} P_{12}^{2}}{\varepsilon}, \\
\dot{m}_{1}= & 0.5 m_{2}+1.5 m_{2} P_{11}+0.5 m_{1}^{2} m_{2} \\
& +\frac{2 \gamma^{2} P_{11}}{\varepsilon}\left(\dot{Y}_{1}-m_{1}\right), \\
\dot{m}_{2}= & \frac{2 \gamma^{2} P_{12}}{\varepsilon}\left(\dot{Y}_{1}-m_{1}\right) .
\end{aligned}
$$

1) Consider the stochastic dynamical system associated to a problem of orientation of a monoaxial satellite and the following initial conditions for the state and observations equations: $X_{1}(0)=0.09, X_{2}(0)=0.65, Y_{1}(0)=2$, $Y_{2}(0)=1$, the final time is $T=1 \mathrm{~s}$. The initial conditions for the filtering equations in which case are given by: 
a) For risk-sensitive filtering equations:

$C_{1}(0)=0.92, C_{2}(0)=0.5, Q_{11}(0)=-400, Q_{12}(0)=100$,

$Q_{22}(0)=-850$.

b) For polynomial filtering equations:

$m_{1}(0)=0.92, m_{2}(0)=0.5, P_{11}(0)=10, P_{12}(0)=20$,

$P_{22}(0)=5$.

Table 4 presents comparison between the exponential mean square cost criterion $J$ for the two types of filtering equations; it can be saw, that the $J_{R-S}$ values are the smallest for all values of the intensity parameter $\varepsilon$.

2) Consider the stochastic dynamical system associated to a problem of orientation of a monoaxial satellite and the following initial conditions for the state and observations equations: $X_{1}(0)=0.115, X_{2}(0)=0.073$, $Y_{1}(0)=2, Y_{2}(0)=1$, the final time is $T=1 s$. The initial conditions for the filtering equations in which case are given by:

a) For risk-sensitive filtering equations:

$$
\begin{aligned}
& C_{1}(0)=0.92, C_{2}(0)=0.5, Q_{11}(0)=-4500, \\
& Q_{12}(0)=100, Q_{22}(0)=-8500 .
\end{aligned}
$$

b) For polynomial filtering equations:

$$
\begin{aligned}
& m_{1}(0)=0.92, m_{2}(0)=0.5, P_{11}(0)=1500, \\
& P_{12}(0)=-879.21, P_{22}(0)=1000 .
\end{aligned}
$$

Table 5 presents comparison between the exponential mean square cost criterion $J$ for the two types of filtering equations; it can be saw, that the $J_{R-S}$ values are the smallest for all values of the intensity parameter $\varepsilon$.

The system Equations (24), (25) and (26) is simulated using Simulink in MatLab7. The performance of the designed equations is compared versus the equations of the polynomial filter [1], with respect to the exponential mean-square exponential criterion $J$.

The Figures 4 and 5 show the Error $_{1}$ and Error $_{2}$ which are defined as Error ${ }_{1}=X_{1}(t)-C_{1}(t)$ (in same form for Error $\left._{2}\right)$; and the exponential mean- square cost criterion values.

Table 4. Comparison of mean-square exponential criterion $J(3)$ for $r-s$ filtering equations and polynomial filtering equations.

\begin{tabular}{ccc}
\hline$\varepsilon$ & $J_{R-S}$ & $J_{P_{0} l}$ \\
\hline 0.01 & 0.3239 & 2.0321 \\
0.1 & 0.3232 & 1.1319 \\
1 & 0.3198 & 0.6655 \\
10 & 0.3063 & 0.3319 \\
100 & 0.2800 & 26.8974 \\
\hline
\end{tabular}

Table 5. Comparison of mean-square exponential criterion $J(3)$ for r-s filtering equations and polynomial filtering equations.

\begin{tabular}{ccc}
\hline$\varepsilon$ & $J_{R-S}$ & $J_{P_{0 l}}$ \\
\hline 0.01 & 0.3842 & 0.5895 \\
0.1 & 0.3835 & 0.4287 \\
1 & 0.3691 & 0.4013 \\
10 & 0.2841 & 0.3054 \\
100 & 0.1454 & 0.2454 \\
\hline
\end{tabular}
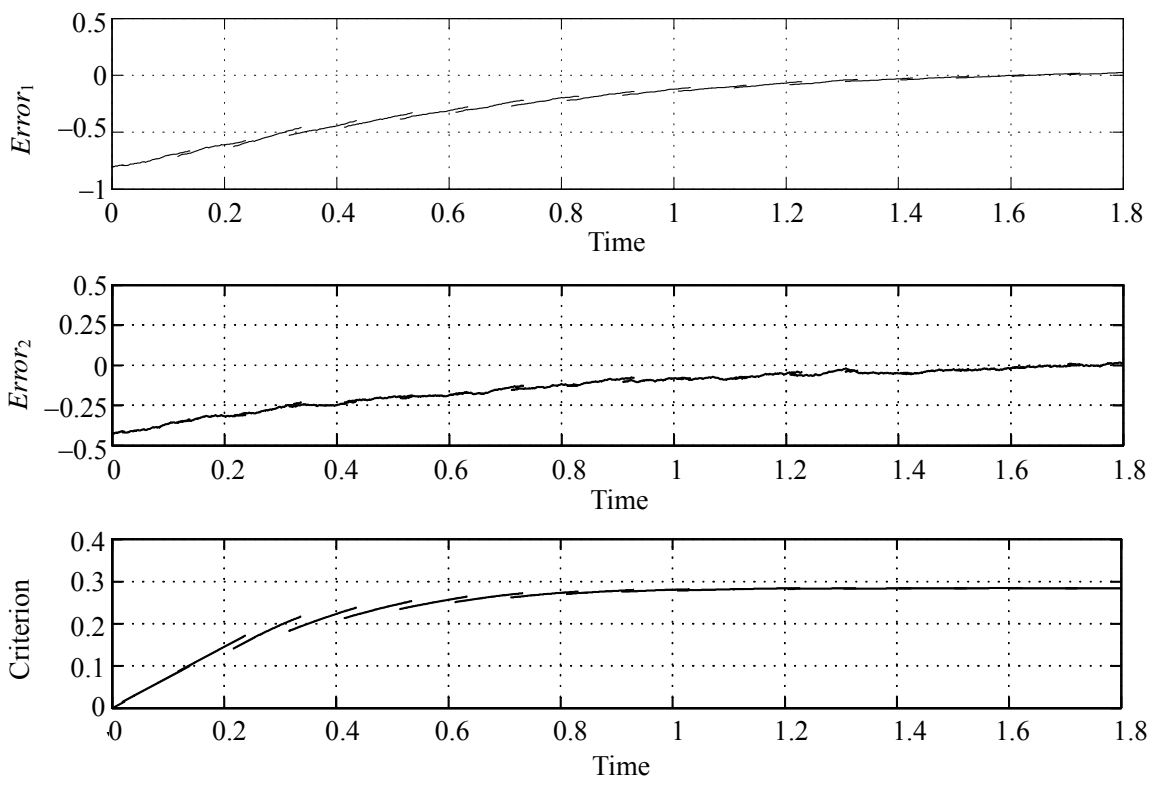

Figure 4. Graphs of the Error $_{1}$, Error ${ }_{2}$, and exponential mean square cost criterion corresponding to the risk-sensitive optimal filtering equations for satellite monoaxial for $\varepsilon=10, X_{1}(0)=0.115, X_{2}(0)=0.073, Y_{1}(0)=2, Y_{2}(0)=1$. 

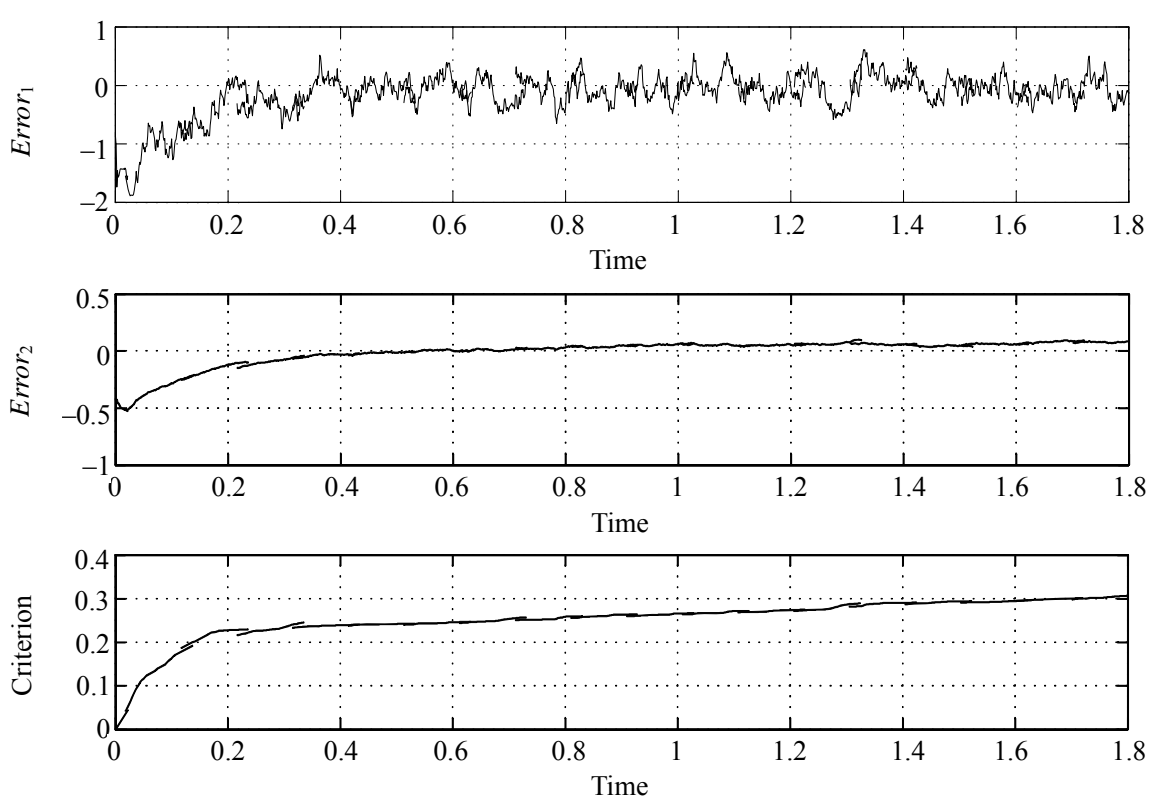

Figure 5. Graphs of the Error $_{1}$, Error ${ }_{2}$, and exponential mean square cost criterion corresponding to the polynomial filtering equations for satellite monoaxial for $\varepsilon=10, X_{1}(0)=0.115, X_{2}(0)=0.073, Y_{1}(0)=2, Y_{2}(0)=1$.

\section{Conclusions}

In this paper the equations have been obtained for the optimal risk-sensitive filtering problem, when the system is polynomial of second and third degree, with presence of Gaussian white noise, exponential mean-square cost criterion to be minimized, with parameter $\varepsilon$ multiplying the Gaussian white noise in the state and observations equations, and taking into account a value function as a viscosity solution of the nonlinear parabolic PDE.

Numerical application is solved for risk-sensitive and polynomial filtering equations for system of second and third degree (and Kalman-Bucy for system of second degree) for some values of parameter $\tilde{\varepsilon}$. The performance for optimal risk-sensitive filtering equations is verified through of the comparison between the values of the exponential mean-square cost criterion $J$ for polynomial and extended Kalman Bucy filtering equations.

It can be seen that the values of the mean square cost criterion $J_{R-S}$ in final time, are smaller than $J_{P o l}$ and $J_{K-B}$ for all values given to the intensity parameter $\varepsilon$.

\section{References}

[1] M. V. Basin and M. A. Alcorta-García, "Optimal Filtering and Control for Third Degree Polynomial Systems," Dynamics of Continuous Discrete and Impulsive Systems, Vol. 10, 2003, pp. 663-680.

[2] M. V. Basin and M. A. Alcorta-García, "Optimal Filtering for Bilinear Systems and Its Application to Terpolymerization Process State," Proceedings of IFAC 13th
Symposium on System Identification, 27-29 August 2003, Rotterdam, 2003, pp. 467-472.

[3] F. L. Lewis, "Applied Optimal Control and Estimation," Prentice Hall PTR, Upper Saddle River, 1992.

[4] V. S. Pugachev and I. N. Sinitsyn, "Stochastic Systems Theory and Applications," World Scientific, Singapore, 2001.

[5] S. S.-T. Yau, "Finite-Dimensional Filters with Nonlinear Drift. I: A Class of Filters including both Kalman-Bucy and Benes Filters," Journal of Mathematical Systems, Estimation, and Control, Vol. 4, 1994, pp. 181-203.

[6] R. E. Mortensen, "Maximum Likelihood Recursive Nonlinear Filtering," Journal of Optimization Theory and Applications, Vol. 2, No. 6, 1968, pp. 386-394. doi:10.1007/ BF00925744

[7] W. M. McEneaney, "Robust $H_{\infty}$ Filtering for Nonlinear Systems," Systems and Control Letters, Vol. 33, No. 5, 1998, pp. 315-325. doi:10.1016/S0167-6911(97)00124-2

[8] M. A. Alcorta-García, M. V. Basin, S. G. Anguiano and J. J. Maldonado, "Sub-optimal Risk-Sensitive Filtering for Third Degree Polynomial Stochastic Systems," IEEE Control Applications \& Intelligent Control, St. Petersburg, 8-10 July 2009, pp. 285-289.

[9] H. K. Khalil, "Nonlinear Systems," 3rd Edition, Prentice Hall, Upper Saddle River, 2002.

[10] W. H. Fleming and W. M. McEneaney, "Robust Limits of Risk Sensitive Nonlinear Filters," Mathematics of Control, Signals, and Systems, Vol. 14, No. 1, 2001, pp. 109-142. doi:10.1007/PL00009879

[11] W. M. McEneaney, "Max-Plus Eigenvector Representations for Solution of Nonlinear H1 Problems: Error Analysis," SIAM Journal on Control and Optimization, Vol. 43, 2004, pp. 379-412. doi:10.1137/S0363012902414688 
[12] D. L. Lukes, "Optimal Regulation of Nonlinear Dynamic Systems," SIAM Journal on Control and Optimization, Vol. 7, No. 1, 1969, pp. 75-100. doi:10.1137/0307007

[13] T. Yoshida and K. Loparo, "Quadratic Regulatory Theory for Analitic Nonlinear Systems with Additive Controls," Automatica, Vol. 25, No. 4, 1989, pp. 531-544. doi:10.10 16/0005-1098(89)90096-4

[14] N. N. Ural'ceva, O. A. Ladyzenskaja and V. A. Solon- nikov, "Newblock Linear and Quasi-Linear Equations for Parabolic Type," American Mathematical Society, Providence, 1968.

[15] H. Sira-Ramírez, R. Márquez, F. Rivas-Echeverría and O. Llanes-Santiago, "Automática y Robótica: Control de Sistemas no Lineales,” Pearson, Madrid, 2005.

[16] A. H. Jazwinski, "Stochastic Processes and Filtering Theory," Dover Publications, Inc., New York, 2007. 